\title{
Phase Transformation Behaviors of Sb-rich Chalcogenide Films under Isothermal and Constant Heating Rate Annealings
}

\author{
Po-Kai Chiu ${ }^{a}$, Shih-Feng Tseng ${ }^{a}$, Wen-Tse Hsiao ${ }^{a}$, Chao-Te Lee ${ }^{a}$, Donyau Chiang ${ }^{a, *}$ \\ ${ }^{a}$ Instrument Technology Research Center, National Applied Research Laboratories, Hsinchu Science Park, Hsinchu, \\ 30076, Taiwan
}

*Corresponding Author: dony@itrc.narl.org.tw

\begin{abstract}
The transformation behaviors of Sb-rich chalcogenide alloys with composition ratio of element group 14 to $\mathrm{Sb}$ ranged from 0.46 to 0.17 were investigated. The constant heating rate and isothermal annealings are applied to evaluate the transformation. The crystallization temperature at both annealing measurements increases with the ratio of element group 14 to $\mathrm{Sb}$ decrease. The activation energies determined from both annealing methods are similar. The Avrami exponents, ranged between 1.7 and 2.4, are extra values obtained from the isothermal measurements. The 2-D growth dominant mechanism is implied by the decided Avrami exponent values. $\mathrm{Sb}$ rhombohedral and $\mathrm{Sb}_{7} \mathrm{Te}$ are the major crystallized phases observed for four compositions and SnTe and InTe phases are mixed among the major phase matrix when samples are annealed at $250^{\circ} \mathrm{C}$ for $30 \mathrm{~min}$. The phase changed alloys have potential applications in the thermal sensor materials beside in the optical and electrical memory materials.
\end{abstract}

Keywords: phase transformation, constant heating rate annealing, isothermal annealing, Sb-rich chalcogenide film, activation energy, Avrami exponent.

\section{Introduction}

Phase-changed (PC) materials are extensively used in rewritable high-density recording media, particularly in optical storage media, such as DVD-RAM, DVD $\pm R W$ and BD-RE, and non-volatile memory media, such as PC random access memory (PC-RAM). Data information is stored in nano-sized scale in films or cells and retrieved by detecting the optical or electrical property differences between the amorphous and crystalline phases ${ }^{(1,2)}$. The phase transformation of the PC materials is a thermal activated process. The optical constants and electrical resistance change with time at temperatures close to their crystallization temperatures. The chalcogenide alloys used in PC memory are robust in extreme harsh environments, especially in the cold or the radiated environments ${ }^{(3,4)}$. This material characteristic provides a potential sensing application to monitor the changes of the optical reflectance and transmittance or electrical resistance with time at an elevated temperature in the harsh environments, such as in aerospace or in nuclear plants.

Two categories of the chalcogenide materials are commonly served with different crystallization behaviors. One group (GST) is located on or near by the tie line of $\mathrm{GeTe}^{-} \mathrm{Sb}_{2} \mathrm{Te}_{3}$ pseudo-binary compounds behaved nucleation-dominated crystallization characteristic. Another group (eutectic) is Sb-Te binary compounds with small amount of Ge, In, Sn or/and other element additives and possesses growth-dominated crystallization characteristic ${ }^{(5)}$. Most of the researches are focused on the first group.

The phase transformation studies of PC materials are extensive $^{(6-11)}$. There are two methods used to evaluate the phase transformation behaviors. One is the constant heating rate annealing, and the other is isothermal annealing. However, most of the explore techniques are to monitor the physical property differences varied with temperature at the constant heating rates between amorphous and crystalline phase states. The physical properties include thermal enthalpy $^{(6,8-10)}$, electrical resistivity ${ }^{(7,11)}$, and optical reflectance $^{(7,10)}$ or optical transmittance. The constant heating rate technique is relatively simple and easily operated but the activation energy of the transition process 
is the only retrieved value to describe the entire process. Few physical phenomena are retrieved and understood from this activation energy. Correspondingly, two parameters, including the activation energy and the Avrami exponent are retrieved from the isothermal annealing measurements using the Johnson-Mehl-Avrami (JMA) formula. The growth mechanisms of the amorphous phase of the PC materials are determined by the Avrami exponent values ${ }^{(12)}$. However, no comparison data between both methods are in a published document within the authors' knowledge.

In this study, we examine the phase transition kinetics of the eutectic alloys of $\mathrm{Sb}$ composition from 48 to 70.8 atomic percent under isothermal annealing and constant heating rate annealing and find the effect of $\mathrm{Sb}$ to $(\mathrm{Sn}+\mathrm{Ge})$ ratio on the crystallization behaviors.

\section{Experimental}

The chemical compositions of four sputtering targets are given in Table 1 in atomic ratio, denoted as $\mathrm{S}-1, \mathrm{~S}-2, \mathrm{~S}-3$ and S-4 for short. The composition of the sputtered films was assumed to be the same as that of the targets. The ratio of $(\mathrm{Sn}+\mathrm{Ge})$ to $\mathrm{Sb}$ decreases with increasing $\mathrm{Sb}$ content to examine the effect of $\mathrm{Sb}$ concentration on the crystallization behaviors. Elements Ge and Sn are the group 14 members in the periodical table. The sputtering condition for preparing these four films used in this research was given in Table 2.

Table 1: Chemical compositions in atomic ratio of four sputtering targets used in this research

\begin{tabular}{|c|c|c|c|c|c|c|c|}
\hline & Sb & Sn & Te & In & Ge & Ga & $(\mathbf{S n + G e ) / S b}$ \\
\hline S-1 & 48 & 22 & 12.5 & 11.5 & 0 & 6 & 0.46 \\
\hline S-2 & 60 & 18 & 10 & 6 & 6 & 0 & 0.4 \\
\hline S-3 & 64.5 & 14.5 & 8 & 7 & 6 & 0 & 0.32 \\
\hline S-4 & 70.8 & 10 & 12.2 & 5 & 2 & 0 & 0.17 \\
\hline
\end{tabular}

The constant heating rate annealings on the sample powders were conducted by differential scanning calorimetry (DSC, Perkin-Elmer Pyris 1) at the heating rates of 5, 10, 20 and $50{ }^{\circ} \mathrm{C} / \mathrm{min}$. The sample powder was scraped from the thick film of 5 to $7 \mu \mathrm{m}$ thickness deposited on a glass substrate. The deposition condition was the same as that described in Table 2 but the deposition process was interrupted every $40 \mathrm{~s}$ and a $3 \mathrm{~s}$ cooling period was added to prevent the film from overheating. A sharp knife blade was applied to scrape the film material on the glass surface and scraped powder was collected. The sample powder sealed inside aluminum pan in DSC was heated from ambient temperature to pre-determined temperatures under the $\mathrm{N}_{2}$ protective environment.

Table 2: sputtering parameters to prepare the deposited film

\begin{tabular}{|l|l|}
\hline Sputtering system & Unaxis DVD Sprinter \\
\hline Substrate & $\begin{array}{l}\text { Glass substrates of 5-inch panel for DSC } \\
\text { and XRD measurements } \\
\text { Glass substrate of linch xlinch x1 mm for } \\
\text { isothermal annealing }\end{array}$ \\
\hline DC power & $500 \mathrm{~W}$ \\
\hline Target diameter & $200 \mathrm{~mm} \Phi \times 6 \mathrm{~mm} \mathrm{t}$ \\
\hline Background pressure & $1 \times 10^{-3} \mathrm{mBar}$ \\
\hline Argon flow rate & $30 \mathrm{sccm}$ \\
\hline Substrate temperature & Ambient temperature \\
\hline Film thickness & $\begin{array}{l}5 \sim 7 \mu \mathrm{m} \text { for DSC and XRD measurements } \\
25 \mathrm{~nm} \text { for isothermal annealing }\end{array}$ \\
\hline
\end{tabular}

The sample films of $25 \mathrm{~nm}$ thickness for isothermal annealing were deposited on the glass substrates of size as 1 -inch by 1 -inch by $1.1 \mathrm{~mm}$ and a $25 \mathrm{~nm}$ thick $\mathrm{ZnS}-\mathrm{SiO}_{2}$ film was on the film top to prevent the oxidation. The isothermal annealings were conducted by a home-built transmittance monitoring instrument in the air. A beam splitter divided a light generated from a laser of $660 \mathrm{~nm}$ wavelength into two portions. One portion of light directed to sample film, passed it and measured the intensity by a power sensor. Another portion of light went directly to a power sensor as reference intensity. The laser intensity was less than $0.7 \mathrm{~mW}$ and the spot diameter was around $3 \mathrm{~mm}$ which would not cause severe temperature increase in the sample film. An automatic data acquisition system was installed in the instrument to collect data. The detailed configuration of the instrument was published elsewhere ${ }^{(13)}$. The annealing temperatures applied in each sample were a few degrees lower than the crystallization temperatures determined by the heating rate of $10 \mathrm{k} / \mathrm{min}$. The crystallized fraction is assumed to be proportional to the transmittance difference and given as the equation below.

$$
f(t)=\frac{T(t)-T_{0}}{T_{f}-T_{0}}
$$

where $\mathrm{f}(\mathrm{t})$ is the transformed crystallization fraction, $\mathrm{T}(\mathrm{t})$ is the transmittance of the sample at the time $t, T_{0}$ is the measured transmittance at the initial stage, and $T_{f}$ is the final transmittance reading.

X-ray diffractometer (XRD, PANalytical X'pert Pro MRD) was conducted to determine the crystal phases 
before and after the phase transition. The X-ray was generated from the $\mathrm{Cu}$ target with beam size of $12 \mathrm{~mm}$ by $0.4 \mathrm{~mm}$ and the scanning speed was $3 \% \mathrm{~min}$. The powder was scratched from the thick film deposited on the glass substrate and the film deposition condition was the same as DSC sample powder preparation. The crystallized powder samples were annealed at temperature of $250^{\circ} \mathrm{C}$ lasting for 30 min under $\mathrm{N}_{2}$ environment. The computer searching software with the aid of the JCPDS card was used to identify the crystal structure of the annealed samples.

\section{Results and Discussions}

Fig. 1 shows the DSC traces at heating rates of 5, 10, 20 and $50 \mathrm{k} / \mathrm{min}$ for sample $\mathrm{S} 1$ in the temperature ranges from $50^{\circ} \mathrm{C}$ to $300^{\circ} \mathrm{C}$. The temperatures of the exothermic curve minima increase with increasing the heating rate. The exothermic peaks come from the as-sputtered powder samples to release the latent heat frozen during the deposition. The scanning temperature ranges extend to $450^{\circ} \mathrm{C}$ for $\mathrm{S} 1$ at a heating rate of $10 \mathrm{k} / \mathrm{min}$ to depict the melting and crystallization, as shown in Fig. 2. The crystallization and melting temperatures are $195.88^{\circ} \mathrm{C}$ and $416.77^{\circ} \mathrm{C}$, respectively.

Samples S2, S3 and S4 have the similar DSC reactions as $\mathrm{S} 1$. The crystallization temperatures are slightly higher but the melting temperatures are lower than that of most GST alloys $^{(8,14)}$.

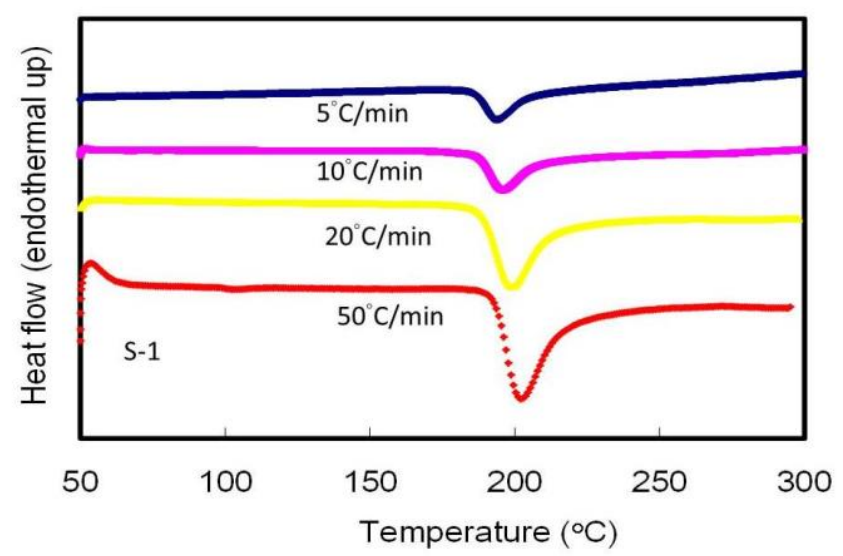

Fig. 1 The DSC traces for sample S1 at 5, 10, 20 and $50^{\circ} \mathrm{C} / \mathrm{min}$ heating rates. The trace minima show exothermic reaction and minima temperatures increase with heating rates.

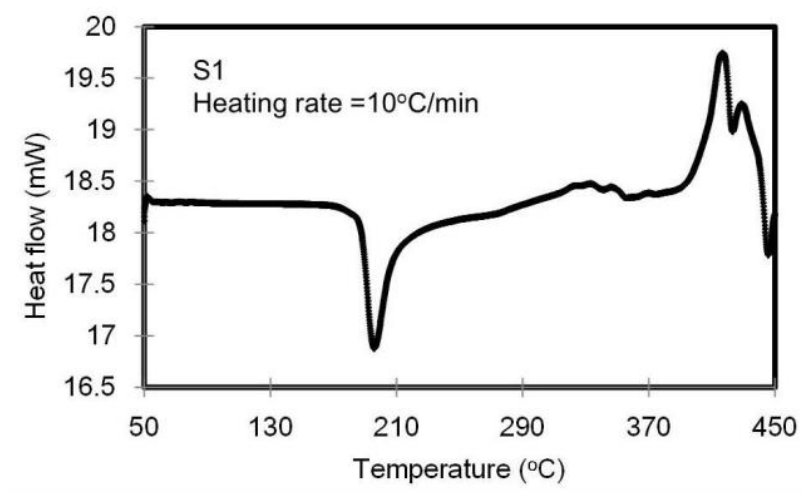

Fig. 2 The scanned DSC temperature ranges are from ambient temperature to $450^{\circ} \mathrm{C}$ to illustrate the whole thermal reactions of the as-sputtered $\mathrm{S} 1$ sample at $10^{\circ} \mathrm{C}$ a minute heating rate

The DSC traces at heating rate of $10^{\circ} \mathrm{C} / \mathrm{min}$ for four samples are given in Fig. 3. The temperature of the first trace minimum increases with increasing the $\mathrm{Sb}$ concentration in alloys. S1 has an exothermic minimum but $\mathrm{S} 2, \mathrm{~S} 3$ and $\mathrm{S} 4$ shows two minima with separated temperature interval decrease. The activation energies determined from the Kissinger's plot are given in Fig. 4 and the energies are 507, 490, 446, and $460 \mathrm{~kJ} /$ mole for samples $\mathrm{S} 1, \mathrm{~S} 2, \mathrm{~S} 3$ and $\mathrm{S} 4$, respectively. The calculated activation energies are double compared to the GST series alloys ${ }^{(8,14)}$.

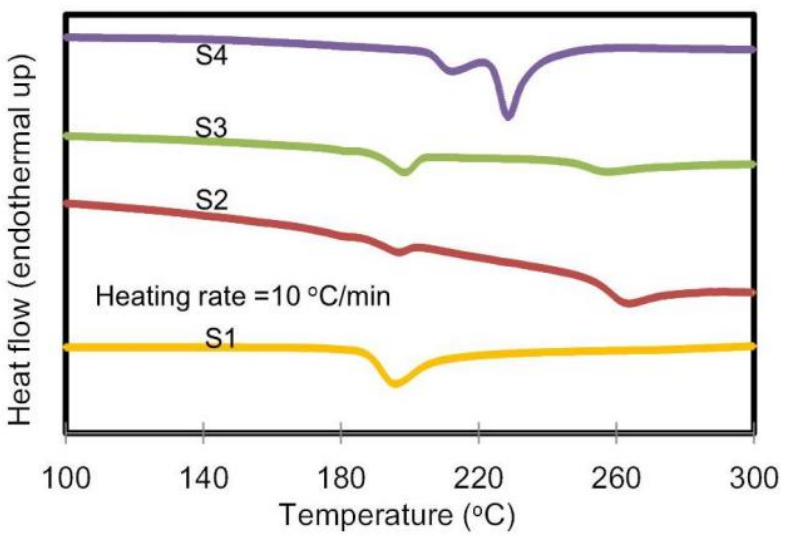

Fig. 3 The DSC traces for samples S1, S2, S3, and S4 at a heating rate of $10^{\circ} \mathrm{C} / \mathrm{min}$.

The activation energy values are relevant to the crystallized phases and mechanisms. The lower activation energies are obtained from the high symmetric crystal structures, such as face center cubic or simple cubic structure and the higher energies are from the less symmetric structures, such as hexagonal or rhombohedral 
structure. The crystallization of GST is nucleation-driven mechanism and that of eutectic alloys is growth-driven mechanism ${ }^{(5)}$.

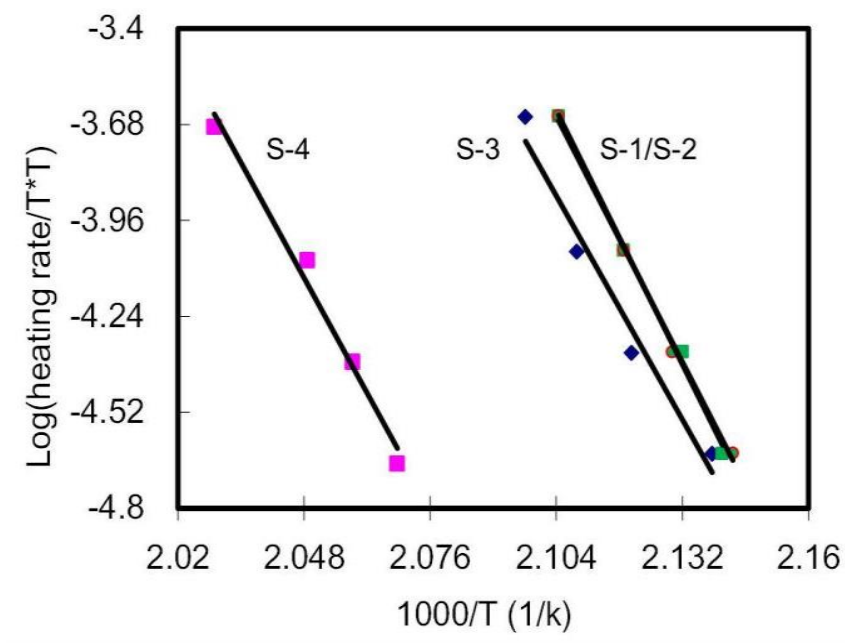

Fig. 4 The Kissinger's plot determines the activation energies for four samples.

The crystallization curves under the isothermal annealings for sample $\mathrm{S} 2$ at different temperatures are shown in Fig. 5. The isothermal annealing temperatures are few degrees lower than that determined from the DSC measurements. The typical $\mathrm{S}$ curves are observed in the diagram with an incubation period, crystallization period, and the saturation period in each curve measured at a fixed temperature. The incubation time increases with decrease of the annealing temperature.

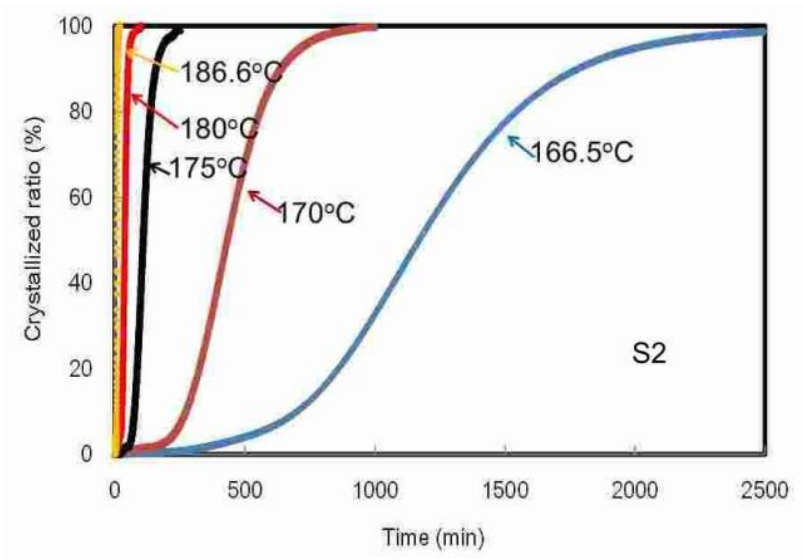

Fig. 5 The isothermal annealing curves of S2 show typical $\mathrm{S}$ shape and the temperatures are few degrees lower than that determined from DSC measurements

Fig. 6 applies the logarithm time scale to clearly illustrate the isothermal annealing treatments. The curves are parallel each other. If the crystallized fraction $\mathrm{f}$ can be described by JMA formula, the effective activation energy and Avrami (time) exponent are decided from the formula. The JMA formula is written as $\mathrm{f}=1-\exp \left[-\mathrm{K}\left(\mathrm{t}-\mathrm{t}_{0}\right)^{\mathrm{n}}\right]$, where $\mathrm{K}=\gamma \exp (-\mathrm{E} / \mathrm{RT})$ and $\gamma, \mathrm{E}, \mathrm{t}, \mathrm{t}_{0}$, and $\mathrm{n}$ are frequency factor, activation energy, isothermal annealing time, incubation time and Avrami exponent, respectively. The similar isothermal crystallized curves are observed for all samples subjected to isothermal annealings.

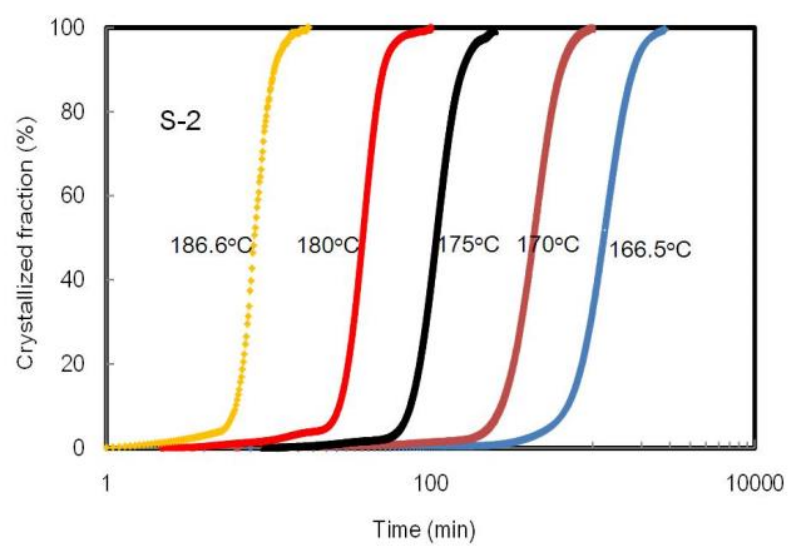

Fig. 6 The same figure as Fig. 5 but the logarithm scale of the abscissa coordinate was used

The Avrami exponent is calculated from the slope of the relation between the $\ln (-\ln (1-\mathrm{f}))$ versus $\ln \left(\mathrm{t}-\mathrm{t}_{0}\right)$, as shown in Fig. 7. The exponents are 2.25, 2.18, 2.02, 1.97, and 2.37 corresponding to temperatures of 186.6, 180.0, $175.0,170.0$, and $166.5^{\circ} \mathrm{C}$, respectively. The crystallized fraction ranges from $10 \%$ to $80 \%$ were calculated because the fractions over $80 \%$ were deviated from the exponential function. The values of $t_{0}$ were taken as the incubation time interval when the crystallized fraction reached $1 \%$ from the beginning. The values $t_{0}$ are necessary to shift the time scale and to obtain the isothermal annealing $S$ curves described satisfactorily by the exponential function. The $t_{0}$ values of S2 under this experimental condition were 5.05, $19.65,53.8,193.7$, and 317.12 minutes for respective annealing temperatures of 186.6, 180.0, 175.0, 170.0, and $166.5^{\circ} \mathrm{C}$. A curve pointed by an arrow in the middle shows the uncorrected $t_{0}$ factor for temperature $180.0^{\circ} \mathrm{C}$ for comparison purpose. The determined Avrami exponent is 5.25 , which is over double value of the correction one and also over the normal expected ranges between 1 and $4^{(12)}$. The measured Avrami exponents for S1, S3, and S4 are 1.71 2.18, 1.96 2.08, and 1.82 2.06 and imply the 2-D 
crystal growth mechanism $^{(12)}$.

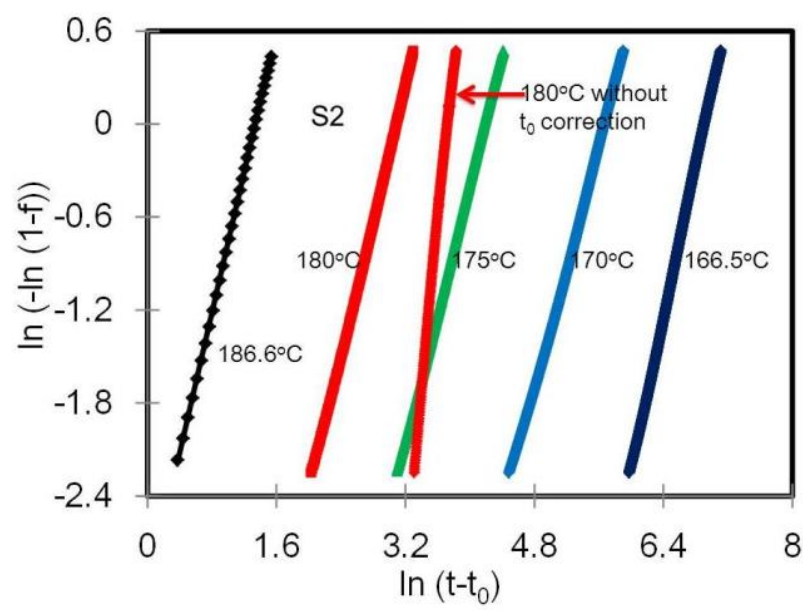

Fig. 7 The Avrami exponent obtained from JMA formula. The $\mathrm{t}_{0}$ correction in abscissa axis is required to shift the isothermal annealing $\mathrm{S}$ curve and to calculate the corrected exponents

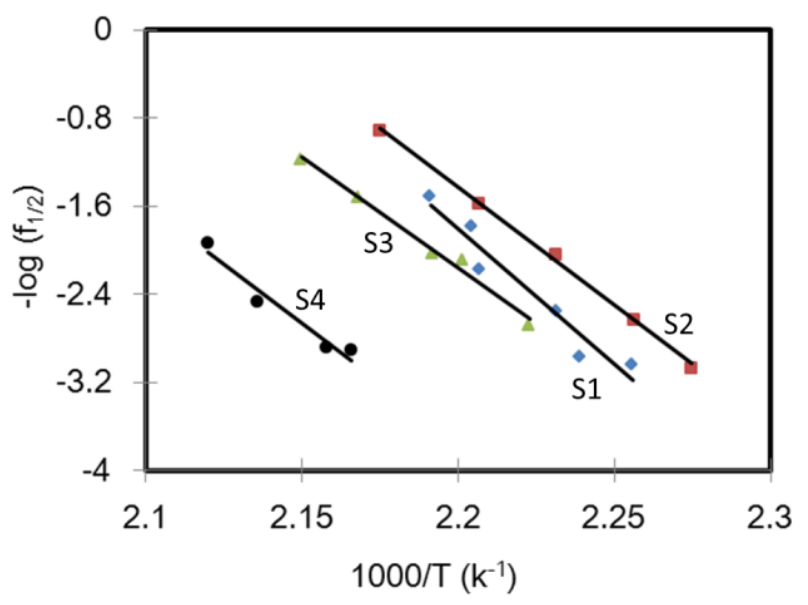

Fig. 8 The activation energies derived from the slopes of relation between the logarithm of time to complete $50 \%$ crystallized fraction versus the reciprocal of absolute temperature

The activation energies derived from the slopes of isothermal annealing treatments are shown in Fig. 8. The ordinate coordinate is the logarithm of time which completes $50 \%$ crystallized fraction, $\mathrm{f}_{1 / 2}$, during the isothermal annealing. The abscissa coordinate is the reciprocal of the absolute temperature. The energies obtained from the regression analysis are 470.1, 411.5, 400.6, and 408.1 kJ/mole for S1, S2, S3, and S4 samples, respectively. The activation energies derived from the isothermal annealings are slightly less than that from the constant heating rate annealings.

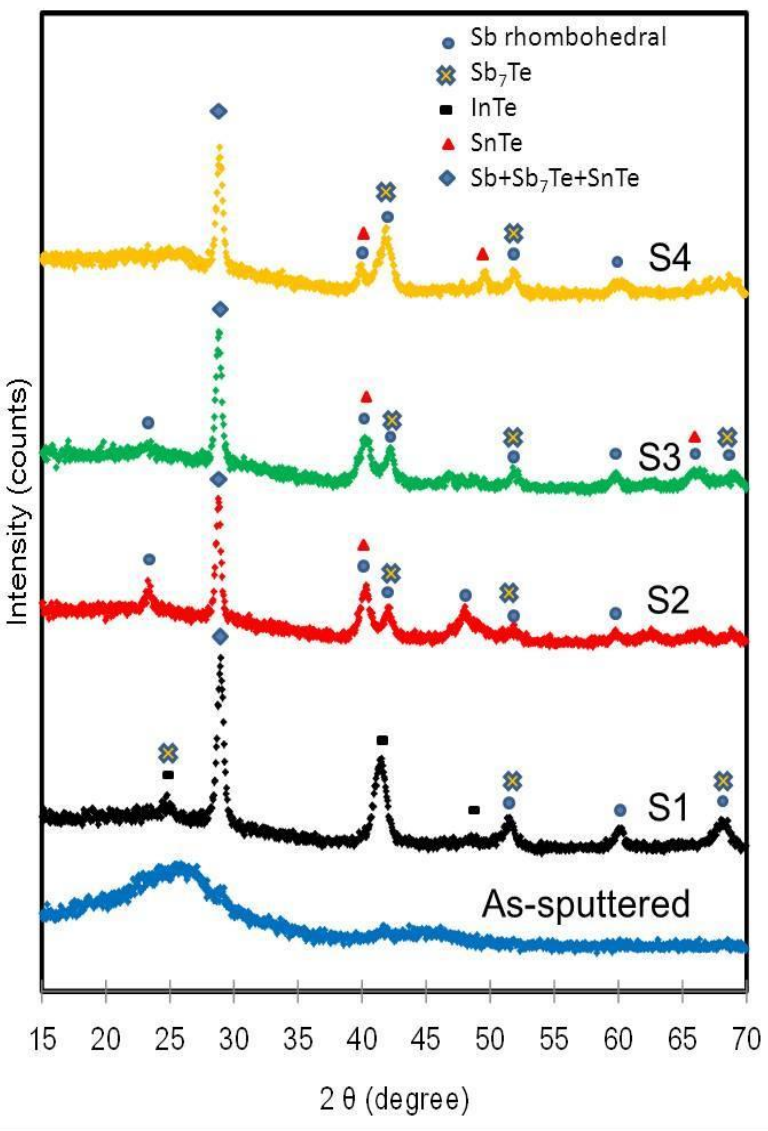

Fig. 9 The phases of as-sputtered and annealed samples determined by XRD. The as-sputtered phase of all samples is amorphous, and the phases of samples annealed at $250^{\circ} \mathrm{C}$ for $30 \mathrm{~min}$ are major $\mathrm{Sb}$ and $\mathrm{Sb}_{7} \mathrm{Te}$ crystals with mixture of few quantities of InTe and SnTe crystalline.

The crystallized phases of four composition samples subjected to $250^{\circ} \mathrm{C}$ for 30 minutes annealing are identified by XRD and Fig. 9 is the result. The bottom curve is amorphous phase obtained from as-sputtered powder of all samples. We apply five symbols, circle, cross, triangle, square, and prism to represent the $\mathrm{Sb}, \mathrm{Sb}_{7} \mathrm{Te}, \mathrm{SnTe}$, InTe, and mixture of $\mathrm{Sb}+\mathrm{Sb}_{7} \mathrm{Te}+\mathrm{SnTe}$ phases. The major crystallized phases are $\mathrm{Sb}$ rhombohedral and $\mathrm{Sb}_{7} \mathrm{Te}$. The other Te compounds are mixed among them. The element $\mathrm{Te}$ is located in the same group as element $\mathrm{O}$ and has the similar electron affinity as $\mathrm{O}$ and which Te compound precipitated out during the annealing is mainly dependent on the exact composition.

\section{Conclusions}


The Sb-rich chalcogenide alloys with composition ratio of element group 14 to $\mathrm{Sb}$ ranged from 0.46 to 0.17 were investigated to illustrate the phase transformation behaviors. The constant heating rate and isothermal annealings are applied to evaluate the transformation. The crystallization temperature at both annealing measurements increases with the ratio of element group 14 to Sb decrease. The activation energy is the only value obtained from the constant heating rate measurements by Kissinger's plot and is roughly a constant, $476 \pm 28 \mathrm{~kJ} /$ mole within the studied composition ranges. Both activation energy and Avrami exponent are two values obtained from the isothermal annealing by Johnson-Mehl -Avrami model. The activation energies are $422.6 \pm 32.0 \mathrm{~kJ} / \mathrm{mole}$ similar to that decided from constant heating rate annealings. The Avrami exponent values are between 1.7 and 2.4, indicating the 2-D growth dominant mechanism. $\mathrm{Sb}$ rhombohedral and $\mathrm{Sb}_{7} \mathrm{Te}$ are the major crystallized phases observed for four compositions and SnTe and InTe phases are mixed among the major phase matrix when samples are annealed at $250^{\circ} \mathrm{C}$ for $30 \mathrm{~min}$. The phase changed alloys have potential applications in the thermal sensor materials beside in the optical and electrical memory materials.

\section{Acknowledgment}

The authors appreciate Mr. Chin-Yen Yeh and Mr. Pang-Chi Liu of CMC Magnetics Corporation for sample preparation and the financial support from Taiwan Science Council under contracts NSC 102-2622-E-492-014-CC3 and MOST 103-2221-E-492-013.

\section{References}

(1) S.R. Ovshinsky : "Reversible electrical switching phenomena in disordered structure", Phys. Rev. Lett. 21(20), pp. 1450-1453, 1968

(2) T. Ohta : "Phase Change Memory: Rewritable Optical Disks and Electronic non-volatile memories", EIPCOS, 22,2004

(3) J. Maimon, K. Hunt, J. Rodgers and L. Burcin : "Results of radiation effects on a chalcogenide nonvolatile memory array", Aerospace Conference Proceedings, vol. 4, pp. 2306-2315, 2004

(4) L-C Wu, B. Liu, Z-T Song, G-M Feng, S-L Feng and B. Chen : "Total Dose Radiation Tolerance of Phase Change Memory Cell with GeSbTe Alloy", Chinese Phys. Lett., Vol. 23(9), pp. 2557-2559, 2006
(5) G. Zhou, H.J. Borg, J.C.N. Rijpers, M.H.R. Lankhorst and J.J. Horikx : "Crystallization behavior of phasechange materials: comparison between nucleation- and growth-dominated crystallization", Proc. SPIE 4090, 108, Optical Data Storage 2000

(6) G. Singh, A. Kaura, M. Mukul, and S.K. Tripathi : "Electrical, optical, and thermal properties of Sn-doped phase change material Ge2Sb2Te5", J. Mater. Sci. 48, pp. 299-303, 2013

(7) H-G Jun, M-H Kwon, D. Kang, D. Lee, and K-B Kim : "New method of evaluating the crystallization activation energy of Ge2Sb2Te5 by in situ resistance measurement”, Jpn. J. Appl. Phys., 50, 020214, 2011

(8) D. Chiang, T-R Jeng, D-R Huang, Y-Y Chang, and C-P Liu : "Kinetic crystallization behavior of phase-change medium”, Jpn. J. Appl. Phys., 38, pp. 1649-1651, 1999

(9) Y. Sutou, T. Kamada, M. Sumiya, Y. Saito, and J. Koide : "Crystallization process and thermal stability of $\mathrm{Ge} 1 \mathrm{Cu} 2 \mathrm{Te} 3$ amorphous thin films for use as phase change materials", Acta Mater., 60, pp. 872-880 2012

(10) Y-C Her, H. Chen, and Y-S Hsu : "Effects of Ag and In addition on the optical properties and crystallization kinetics of eutectic Sb70Te30 phase-change recording film”, J. Appl. Phys., 93, 10097, 2003

(11) Y. Kim, U. Huang, Y.J. Cho, H.M. Park, M-H Cho, P-S Cho, and J-H Lee : "Change in electrical resistance and thermal stability of nitrogen incorporated Ge2Sb2Te5 films”, Appl. Phys. Lett., 90, 021908, 2007

(12) AK Jena, and MC Chaturvedi : "Phase Transformations in Materials", Prentice Hall, pp. 243-247, 1992, ISBN 0-13-663055-3

(13) D. Chiang, S-F Tseng, W-T Hsiao, C-T Lee, and D-R Huang : "The phase transition kinetics of phasechanged materials investigated by a transmittance measurement instrument", MRS-T annual meeting, Oct. 18-19, 2013, Jhongli-city, Taiwan

(14) I. Friedrich, V. Weidenhof, W. Njoroge, P. Franz, and M. Wutting : "Structural transformations of $\mathrm{Ge}_{2} \mathrm{Sb}_{2} \mathrm{Te}_{5}$ films studied by electrical resistance measurements", J. Appl. Phys., 87, pp. 4130-4134, 2000 